\title{
Home Oxygen Evaluation by Respiratory Therapists in Patients Hospitalized for COPD Exacerbations: The RIsOTTO Study
}

\author{
Ai-Yui M Tan, David L Vines, Jerry A Krishnan, Valentin Prieto-Centurion, and \\ Thomas J Kallstrom
}

\begin{abstract}
BACKGROUND: The majority of prescriptions for supplemental oxygen are written when patients are discharged to home from the hospital and the evaluation of these patients is inconsistent. Respiratory Therapists receive training in the evaluation and management of patients needing oxygen. The primary goal of the study was to estimate the frequency with which respiratory therapists (RTs) evaluate the need for home oxygen in patients hospitalized for COPD exacerbations before discharge. METHODS: An online questionnaire was distributed to RTs in the United States by the American Association for Respiratory Care. RTs were asked to indicate how frequently they evaluate the need for home oxygen on an ordinal scale: Never, Rarely/occasionally, Sometimes, Most of the time, Almost every time, or Every time. Consistent evaluation for home oxygen was defined as performing an evaluation for home oxygen therapy Almost every time or Every time (ie, $>75 \%$ of the time). Bivariate and multivariable analyses were assessed using the Fisher exact test and logistic regression models. RESULTS: Of 611 respondents, 490 were eligible for analysis. Fifty-eight percent of RTs reported consistently evaluating patients for home oxygen at rest, whereas $43 \%$ reported doing so during activity and $14 \%$ during sleep. Consistent evaluation for home oxygen requirements at rest was significantly associated with more years of practice $(P=.03$; highest among RTs with $\geq 30 \mathrm{y}$ of practice at $40 \%)$, region of practice $(P=.001$; highest in the Midwest at $44 \%)$, and greater familiarity with criteria for home oxygen $(P<.001$; highest among RTs who selected Very familiar with guidelines from the Centers for Medicare and Medicaid Services at 58\%). Practice in the Midwest and greater familiarity with criteria for home oxygen was associated with consistent evaluation for home oxygen during activity. Practice in the Midwest (vs Northeast; adjusted odds ratio $2.56, P<.001$ ) and being very familiar with home oxygen criteria (vs not at all familiar; adjusted odds ratio 5.66, $P<.001$ ) were independently associated with a higher odds of evaluating for home oxygen at rest and with activity. Only $25 \%$ of RTs were involved in making decisions about home oxygen equipment. CONCLUSIONS: RTs do not consistently evaluate patients hospitalized for COPD exacerbations for home oxygen prior to discharge, and only a minority of RTs are involved in selecting home oxygen equipment. Key words: home oxygen; home oxygen evaluation; COPD; COPD exacerbation; respiratory therapist; hospital to home transition. [Respir Care 2021;66(2):183-190. (C) 2021 Daedalus Enterprises]
\end{abstract}

\section{Introduction}

COPD is a common respiratory disorder that affects 15 million individuals in the United States. COPD is the fourth leading cause of death in the world and is projected to be the third leading cause of death by 2020 (https://goldcopd.org, Accessed July 5, 2020). The majority of prescriptions for supplemental oxygen are written when patients are discharged to home (ie, home oxygen) following an acute cardiopulmonary illness, rather than in the out-patient setting. ${ }^{1,2}$ However, a recent study in subjects hospitalized for COPD exacerbations suggests that the evaluation of patients for home oxygen is inconsistent. ${ }^{3}$ Moreover, patients with COPD report various concerns about home oxygen therapy following hospitalization, including malfunctioning oxygen equipment, lack of education about the use of the equipment, and inadequate access to appropriate home oxygen equipment. ${ }^{4}$ 
Respiratory therapists (RTs) receive training in the evaluation and management of patients with COPD and other lung disorders (https://www.aarc.org, Accessed July 5,

See the Related Editorial on Page 347

2020). ${ }^{5}$ The American Association for Respiratory Care (AARC), the largest professional organization for RTs in the United States, estimates that there are $>170,000$ RTs in the United States alone, working primarily in hospitals and other acute care settings. However, the role of RTs in the evaluation for home oxygen before discharge in patients hospitalized for COPD has not been studied. This study was commissioned by the AARC as part of its efforts to support the U.S. COPD National Action Plan, the first-ever framework for action to reduce the public health impact of COPD in the United States (https://www.aarc.org/copdnational-action-plan, Accessed July 5, 2020).

The primary goal of the Home Oxygen Evaluation by Respiratory Therapists in Patients Hospitalized for COPD Exacerbations (RIsOTTO) Study was to estimate the frequency with which RTs evaluate the need for home oxygen in patients hospitalized for COPD exacerbations at the time of transition from hospital to home. Secondary goals included identification of RT characteristics associated with consistent evaluation for home oxygen therapy and decision makers of selecting home oxygen equipment.

Drs Tan, Krishnan, and Prieto-Centurion are affiliated with the Division of Pulmonary, Critical Care, Sleep and Allergy, University of Illinois at Chicago, Chicago, Illinois. Dr Vines is affiliated with the Department of Cardiopulmonary Sciences, Division of Respiratory Care, Rush University Medical Center, Chicago, Illinois. Dr Krishnan is affiliated with the Population Health Sciences Program, University of Illinois Hospital \& Health Sciences System, Chicago, Illinois. Mr Kallstrom is affiliated with the American Association for Respiratory Care, Irving, Texas.

Dr Tan presented a version of this paper at the American Thoracic Society 2017, held May 19-24, 2017, in Washington, DC.

Supplementary material related to this paper is available at http://www. rcjournal.com.

Dr Tan has disclosed a relationship with the National Institutes of Health (NIH). Dr Krishnan has disclosed relationships with the NIH, the PatientCentered Outcomes Research Institute, ResMed, and Regeneron. Dr Vines has disclosed a relationship with Ohio Medical. Dr PrietoCenturion has disclosed relationships with the NIH and ResMed. $\mathrm{Mr}$ Kallstrom is Executive Director of the American Association for Respiratory Care and Publisher of ResPiRATORY CARE.

Correspondence: Ai-Yui M Tan MD, Loyola University Medical Center, Division of Pulmonary and Critical Care, 2160 S 1st Ave, Maywood, IL, 60153. E-mail: Ai-Yui.Tan@lumc.edu.

DOI: $10.4187 /$ respcare. 07628

\section{QUICK LOOK}

\section{Current knowledge}

Home oxygen therapy is a significant burden, and the evaluation for home oxygen needs in patients hospitalized with COPD is not adequate. The role of respiratory therapists (RTs) in assessing home oxygen needs prior to hospital discharge in patients hospitalized with COPD has not been studied.

\section{What this paper contributes to our knowledge}

A cross-sectional study among RTs who are members of the AARC indicates that RTs do not consistently evaluate home oxygen needs in hospitalized patients with COPD and are rarely involved in selecting home oxygen equipment at the time of hospital-to-home transition. Less than half of surveyed RTs (42\%) were very familiar with the Centers for Medicare and Medicaid Services criteria for home oxygen.

\section{Methods}

\section{Study Population}

The AARC invited its RT membership to participate in the RIsOTTO study. Questionnaire respondents were eligible to participate in the study if they were respiratory therapists, provided care for patients hospitalized for COPD exacerbations, worked in acute care settings, and practiced in the United States. Respondents did not receive compensation for participation in the study. The study was considered exempt from human subjects review by the Institutional Review Boards at the University of Illinois at Chicago (\#2016-0936) and Rush University Medical Center in Chicago (\#16112301-IRB01).

\section{Questionnaire Domains}

The study questionnaire included 3 domains: characteristics of RTs, evaluation for home oxygen therapy, and selection of home oxygen equipment. The questionnaire can be viewed in the supplementary materials at http://www.rcjournal.com.

Characteristics of RTs. Based on the AARC Human Resource Study (https://www.aarc.org/resources/toolssoftware/aarc-respiratory-therapist-human-resource-study2014/ Accessed November 5, 2020), respondents were asked to report their highest level of formal education (associate's degree, bachelor's degree, master's degree, $\mathrm{PhD}$, or other); credentials (registered respiratory therapist, 


\section{Home Oxygen Evaluation by RTs}

certified respiratory therapist, certified pulmonary function technologist, registered pulmonary function technologist, other); years of practice (ie, $<10 \mathrm{y}, 10-19 \mathrm{y}, 20-29 \mathrm{y}$, or $\geq$ $30 \mathrm{y}$ ); and practice location (ie, name of state). We also asked respondents to report their degree of familiarity with U.S. Centers for Medicare and Medicaid Services (CMS) criteria for home oxygen (ie, Very, Somewhat, Not at all familiar; https://www.cms.gov/medicare-coverage-database/ details/ncd-details.aspx?NCDId=169, Accessed July 5, 2020).

Evaluation for Home Oxygen. In the United States, CMS criteria for coverage of home oxygen include the presence of severe lung disease or hypoxia-related symptoms that might be expected to improve with oxygen therapy; qualifying room air arterial blood gas or $\mathrm{S}_{\mathrm{pO}_{2}}$ results at rest, during activity, or during sleep; and that these clinical tests be obtained no earlier than $2 \mathrm{~d}$ prior to hospital discharge. ${ }^{6} \mathrm{We}$ therefore asked RTs to report the frequency with which they evaluated patients hospitalized for COPD exacerbations for home oxygen according to these CMS criteria: Never, Rarely/occasionally (ie, $1-25 \%$ of the time), Sometimes (ie, $26-50 \%$ of the time), Most of the time (ie, $51-75 \%$ of the time), Almost every time (ie, $76-95 \%$ of the time), or Every time ( $>95 \%$ of the time). The question was repeated 3 times to assess the frequency of evaluation at rest, during activity, and during sleep.

Selection of Home Oxygen Equipment. RTs were asked to identify individuals in their institution who make decisions regarding selection of types of home oxygen equipment; options included RT, registered nurse, social worker, physician, durable medical equipment company, I do not know, and Other). Respondents had the option to select $>1$ of these categories.

\section{Questionnaire Administration}

The RIsOTTO questionnaire was piloted among 4 RTs at 1 institution and reviewed for content and clarity prior to data collection. The AARC sent a newsletter via e-mail to all members in October 2016 with an invitation that included a link to the online questionnaire (SurveyMonkey, San Mateo, California). Two reminder e-mails were sent 2 and 5 weeks after the initial e-mail. The questionnaire was closed in February 2017. Response rate was determined by counting unique internet provider addresses, with duplicate addresses removed before analysis. The results of the pilot questions were not included in the final analyses.

\section{Analysis}

Responses were expressed as a frequency and proportion of participants. In the analyses, we combined some response options to simplify interpretation. For the characteristics of RTs, we presented the highest level of education as bachelor's degree or higher versus less than bachelor's degree, and credentials as registered respiratory therapist, certified respiratory therapist, or other. Responses regarding the state in which RTs practiced were re-classified into regions (ie, Midwest, Northeast, South, or West), as defined by the U.S. Census Bureau (https://www2.census.gov/geo/pdfs/maps-data/maps/ reference/us_regdiv, Accessed July 5, 2020). If participants listed more than one state in their answer, they were assigned to the U.S. region of the first state listed. Differences in RT characteristics between respondents from the Midwest and other U.S. regions (ie, Northeast, South, or West) were evaluated with chi-square tests. The frequency of evaluation for home oxygen was presented as a dichotomous variable, defined as consistently ( $>75 \%$ of the time) versus not consistently.

We examined the association between baseline characteristics of RTs and the frequency of consistent evaluation for home oxygen in bivariate (Fisher exact tests) and multivariate (logistic regression models) analyses. RT characteristics that were significantly $(P<.05)$ associated with evaluation for home oxygen in bivariate analyses were included in the multivariate logistic regression models to identify independent predictors of consistent evaluation. These results were presented as adjusted odds ratios with 95\% CIs. A 2 -sided $P$ value $<.05$ was used to define a statistically significant difference. Analyses were performed using Stata 14 (StataCorp, College Station, Texas) and SAS 9.4 (SAS Institute, Cary, North Carolina).

\section{Results}

\section{RT Characteristics}

Of 611 respondents, 490 (79\%) were included in the analyses. Four duplicate responses (based on internet provider addresses) were excluded. The most common reasons for ineligibility were not working in acute care settings $(n=51,8 \%)$ and not providing care for patients hospitalized for COPD ( $n=47,8 \%$; see the supplementary materials at http://www.rcjournal.com). The study participants were recruited from 49 of 50 states in the United States (see the supplementary materials at http://www.rcjournal.com).

Just over half (53\%) of RTs had a bachelor's degree or higher (Table 1). A majority (85\%) were credentialed as registered respiratory therapists. More than a third (36\%) of RTs had at least $30 \mathrm{y}$ of work experience. RTs worked in all regions in the United States, most commonly in the Midwest (36\%) and least commonly in the West (17\%). Only 2 participants reported that they practiced in $>1$ state in different U.S. regions. There were no significant differences between RTs from the Midwest compared to the other U.S. regions 
Table 1. Respondent Characteristics

\begin{tabular}{lc}
\hline \hline Education & \\
Bachelor's degree or higher & $262(53)$ \\
Less than bachelor's degree & $223(46)$ \\
Not reported & $5(1)$ \\
Credentials & \\
$\quad$ Registered respiratory therapist & $418(85)$ \\
Certified respiratory therapist & $49(10)$ \\
Other credentials* & $22(4)$ \\
Not reported & $1(<1)$ \\
Years of practice & \\
$<10$ & $99(20)$ \\
10-19 & $92(19)$ \\
$20-29$ & $123(25)$ \\
$\geq 30$ & $176(36)$ \\
Not reported & $0(0)$ \\
Region of practice & \\
Midwest & $178(36)$ \\
Northeast & $104(21)$ \\
South & $124(25)$ \\
West & $84(17)$ \\
Not reported & $0(0)$ \\
Familiarity with CMS home oxygen criteria & \\
Very familiar & $206(42)$ \\
Somewhat familiar & $211(43)$ \\
Not at all familiar & $36(7)$ \\
Not reported & $37(8)$ \\
\hline Data are presented as $n$ (\%). $N=490$. & \\
* Other credentials include adult critical care specialty, asthma educator certification, bachelor's \\
of science in respiratory therapy, certified pulmonary function technologist, case manager, emer- \\
gency medical technician, licensed respiratory therapist, neonatal pediatric respiratory care spe- \\
cialist, registered pulmonary function technologist. \\
CMS = Centers for Medicare and Medicaid Services \\
\hline \\
\hline
\end{tabular}

with regard to education, credentials, years of practice, or familiarity with the CMS home oxygen criteria (see the supplementary materials at http://www.rcjournal.com). Fewer than half (42\%) of all RTs reported being very familiar with CMS criteria for home oxygen.

\section{Evaluation for Home Oxygen Associated With RT Characteristics}

RTs indicated that they consistently (ie, $>75 \%$ of the time) performed evaluation for the need of home oxygen in patients in the $48 \mathrm{~h}$ prior to hospital discharge in just over half $(58 \%)$ of patients at rest and in a minority of patients during activity or sleep (43\% and $14 \%$, respectively; Fig. 1).

In bivariate analyses, consistent evaluation for home oxygen at rest was significantly associated with more years of practice $(P=.03)$, region of practice $(P=.001$; highest in the Midwest), and greater familiarity with CMS home oxygen criteria $(P<.001)$ (Table 2$)$. A similar pattern was noted for RT characteristics associated with consistent

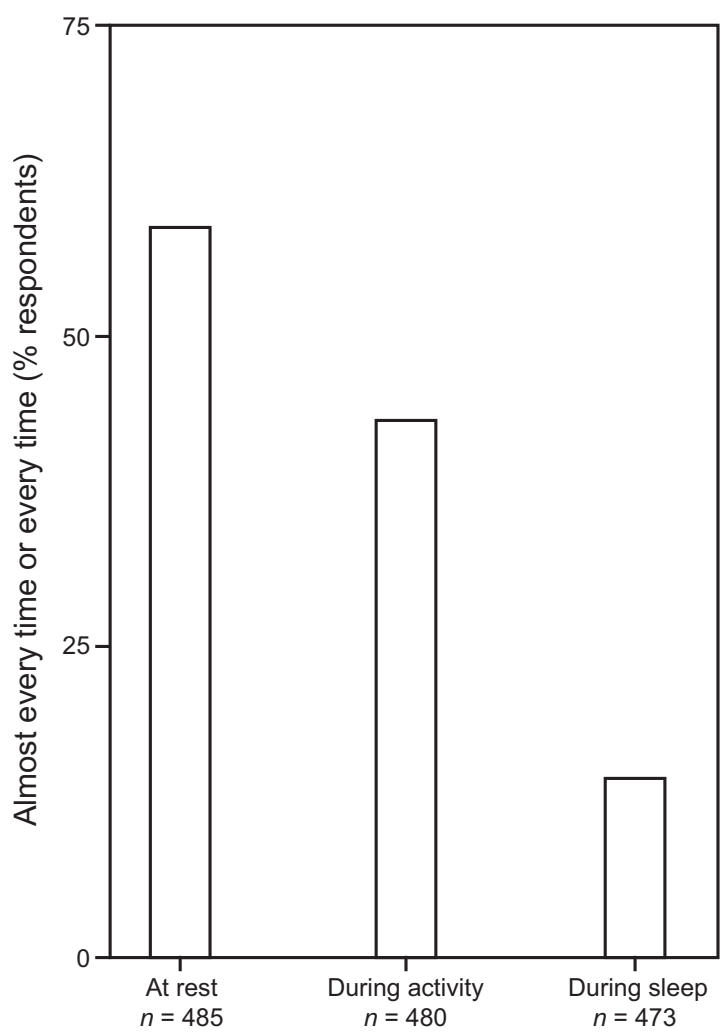

Fig. 1. Respiratory therapist responses as to how consistently they evaluate the need for home oxygen therapy before patient discharge. Also shown are the number of respondents who answered in each patient evaluation scenario (at rest, during activity, and during sleep; $N=490$ ).

evaluation with activity, but there was no significant association with the number of years of practice $(P=.10)$. None of the RT characteristics we examined were associated with consistent evaluation during sleep.

Multivariable analyses indicate that practice in the Midwest (vs practice in the Northeast) was independently associated with higher adjusted odds ratios of consistently evaluating for home oxygen at rest and during activity of 2.56 (95\% CI 1.43-4.58, $P=.001)$ and 2.52 (95\% CI 1.42 $4.50, P=.002$ ), respectively (Figure 2). Similarly, being very familiar with CMS home oxygen criteria (vs not at all familiar) was associated with higher adjusted odds of consistently evaluating the need for home oxygen at rest and during activity, but not during sleep (adjusted odds ratio 5.66 [95\% CI 2.56-12.48, $P<.001$ ], 5.37 [95\% CI 2.2013.10, $P<.001]$, and 2.26 [95\% CI 0.73-7.01, $P=.16]$, respectively).

\section{Selection of Home Oxygen Equipment}

Only $25 \%$ of RTs reported being involved in selecting the home oxygen equipment for patients before discharge, including $3 \%$ reporting joint decisions with physicians and 3\% 


\section{Home OXYgen Evaluation by RTs}

Table 2. Consistent Evaluation for Home Oxygen and Association With RT Characteristics

\begin{tabular}{|c|c|c|c|}
\hline & At Rest & With Activity & During Sleep \\
\hline \multicolumn{4}{|l|}{ Education } \\
\hline Bachelor's degree or higher & $146(52)$ & $103(50)$ & $33(49)$ \\
\hline Less than bachelor's degree & $135(48)$ & $102(50)$ & $34(51)$ \\
\hline$P$ & .31 & .14 & .43 \\
\hline \multicolumn{4}{|c|}{ Credentials } \\
\hline Registered respiratory therapist & $243(86)$ & $176(85)$ & $56(84)$ \\
\hline Certified respiratory therapist & $28(10)$ & $22(11)$ & $8(12)$ \\
\hline Other & $12(4)$ & $9(4)$ & $3(4)$ \\
\hline$P$ & .94 & .92 & .80 \\
\hline \multicolumn{4}{|l|}{ Years of practice } \\
\hline$<10$ & $53(19)$ & $35(17)$ & $19(28)$ \\
\hline $10-19$ & $42(15)$ & $33(16)$ & $12(18)$ \\
\hline $20-29$ & $75(26)$ & $57(27)$ & $16(24)$ \\
\hline$\geq 30$ & $114(40)$ & $83(40)$ & $21(31)$ \\
\hline$P$ & .03 & .10 & .44 \\
\hline \multicolumn{4}{|l|}{ Region of practice } \\
\hline Midwest & $125(44)$ & $96(46)$ & $25(37)$ \\
\hline Northeast & 47 (17) & $31(15)$ & $13(19)$ \\
\hline South & $66(23)$ & $48(23)$ & $15(22)$ \\
\hline West & $46(16)$ & $33(16)$ & $15(22)$ \\
\hline$P$ & .001 & .001 & .71 \\
\hline \multicolumn{4}{|l|}{$\begin{array}{l}\text { Familiarity with CMS home } \\
\text { oxygen criteria }\end{array}$} \\
\hline Very familiar & $157(58)$ & $124(62)$ & $38(58)$ \\
\hline Somewhat familiar & $102(38)$ & $70(35)$ & $24(36)$ \\
\hline Not at all familiar & $12(4)$ & $7(3)$ & $4(6)$ \\
\hline$P$ & $<.001$ & $<.001$ & .12 \\
\hline \multicolumn{4}{|c|}{$\begin{array}{l}\text { Data are presented as } n \text { (\%). In bivariate analyses, region of practice (Midwest) and greater fa- } \\
\text { miliarity (very familiar) with CMS home oxygen criteria were associated with consistent evalua- } \\
\text { tion for home oxygen at rest and with activity. More years of practice }(\geq 30 \mathrm{y}) \text { was associated } \\
\text { with consistent evaluation for home oxygen at rest. } \\
\text { CMS = Centers for Medicare and Medicaid Services }\end{array}$} \\
\hline
\end{tabular}

reporting joint decisions with others (Figure 3). In $18 \%$ of cases, respondents indicated that durable medical equipment companies made decisions about home oxygen equipment.

\section{Discussion}

In this national study of RTs conducted by the AARC, we found that RTs do not consistently evaluate patients hospitalized for COPD exacerbations for home oxygen prior to hospital discharge. RTs who practice in the Midwest and who report being very familiar with CMS criteria for home oxygen were more likely to consistently evaluate patients for home oxygen at rest and with activity. Also, only a minority of RTs are involved in selecting home oxygen equipment prior to hospital discharge.

To our knowledge, the RIsOTTO study is the first study to examine the role of RTs in assessing the need for home oxygen in patients hospitalized for COPD exacerbation.
Previous studies have focused on RT practices regarding noninvasive ventilation, tracheostomy decannulation, and airway clearance. ${ }^{7,8}$ RT training requires $2-4$ y of formal education at accredited respiratory therapy programs (https://www.aarc.org/education/educator-resources/findan-accredited-respiratory-care-program, Accessed July 5,2020 ) in the evaluation and management of patients with lung diseases and in the use of mechanical ventilation and associated devices and equipment (eg, nebulizers, inhalers, ventilators, oxygen equipment). RTs also play an essential role in educating patients on proper management of their home oxygen equipment. However, only $58 \%$ of RTs reported consistently (ie, $>75 \%$ of the time) evaluating hospitalized patients for home oxygen at rest, $43 \%$ during activity, and only $14 \%$ during sleep. The low proportion of RTs evaluating patients for home oxygen at rest was disappointing given the well-known survival benefits of home oxygen in patients with COPD and severe hypoxemia. ${ }^{9,10}$ Moreover, studies also suggest that supplemental oxygen improves dyspnea and exercise tolerance in patients with hypoxemia during physical activity. ${ }^{11-13}$ While it is possible that evaluation for home oxygen is performed by other clinicians besides RTs (eg, nurses, medical residents), a previous study in 2 hospitals indicates that gaps in evaluation for home oxygen are common. ${ }^{3}$ In this previous study, only $22 \%$ (73 of 335) of subjects hospitalized with COPD had an adequate evaluation for home oxygen and only $16 \%$ (54 of 335) had adequate documentation of home oxygen requirements.

Recent reports have described that clinicians feel mostly unprepared when addressing patient concerns about home oxygen therapy. ${ }^{4,6}$ In a study of 507 subjects with COPD on home oxygen therapy, subjects reported different types of home oxygen equipment (eg, stationary concentrator, portable gas tanks, oxygen-conserving device, oxygen refill system, liquid oxygen, portable concentrator). ${ }^{14}$ Participants reported one or more of these types of home oxygen equipment in 25 different combinations (eg, stationary concentrator plus gas tanks; stationary concentrator plus gas tanks with oxygen conserving device; liquid oxygen only). Home oxygen equipment should be tailored to the patient's needs based on their need for oxygen flow (eg, $2 \mathrm{~L} / \mathrm{min}$ continuously vs $4 \mathrm{~L} / \mathrm{min}$ continuously), length of time they expect to be out of the home per day, and ability to lift or carry oxygen equipment. These issues can complicate the evaluation of a patient for home oxygen and providing adequate patient education about home oxygen, especially if the same home oxygen equipment is not available in the hospital setting prior to discharge.

The variability in the evaluation for home oxygen by region of practice among RTs is unexplained. Among RTs in the RIsOTTO study, education, credentials, and years of practice did not differ significantly across U.S. regions. Also, according to the AARC, training requirements do not differ 


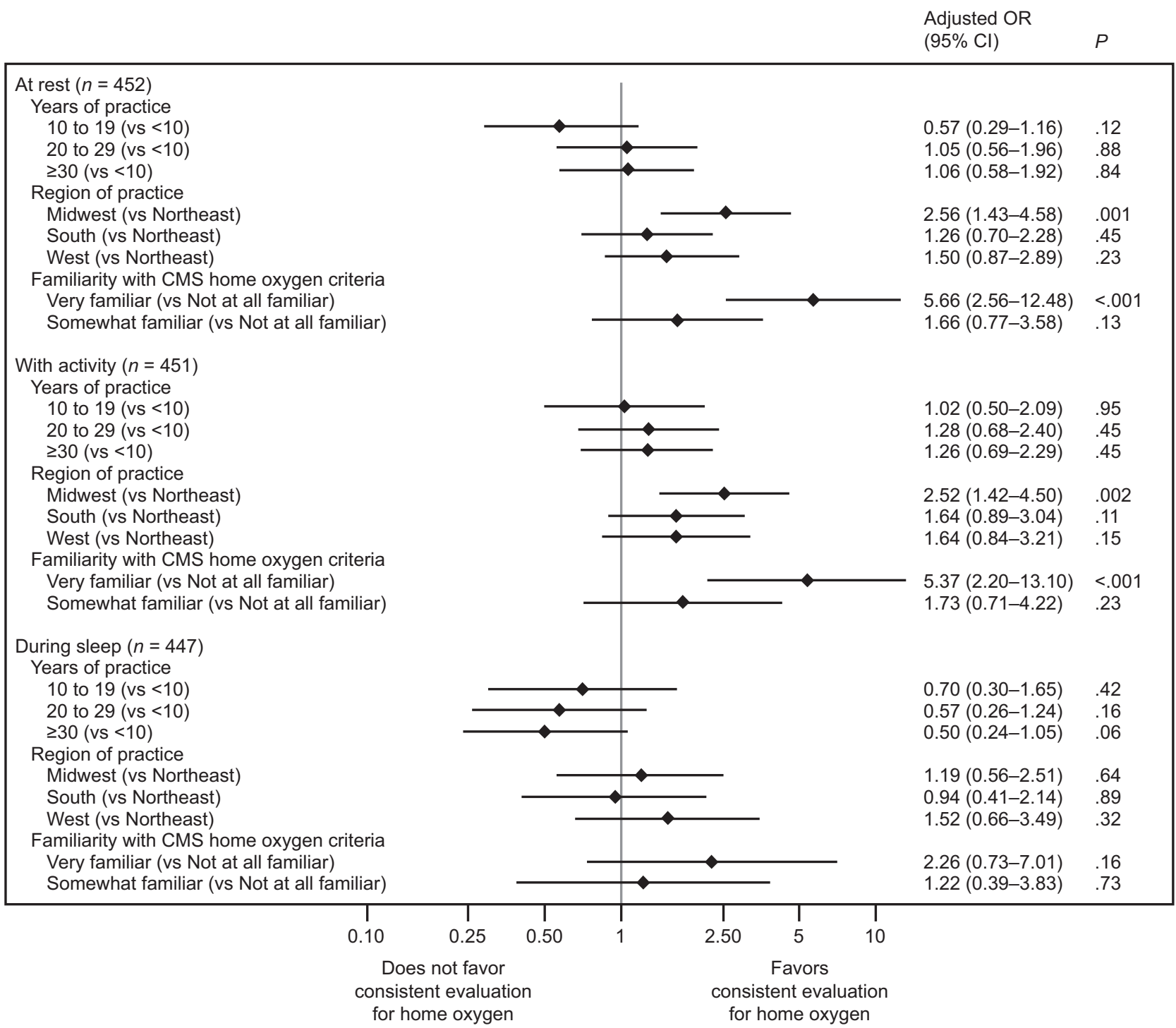

Fig. 2. Forest plot. Multivariable analyses adjusted for years of practice, region of practice, and familiarity with CMS home oxygen criteria. Results of these analyses indicate that practice in the Midwest (vs. practice in the Northeast) and being Very familiar with CMS criteria for home oxygen (vs. Not at all familiar) were independently associated with higher odds of consistently evaluating for home oxygen at rest and with activity. $\mathrm{CMS}=$ Centers for Medicare and Medicaid Services.

significantly across the United States. Regional variation in care by physicians and nurses across the United States has been well documented and may relate to differences in reimbursement, state regulations, and other factors (https://www. dartmouthatlas.org, Accessed July 5, 2020). However, we are not aware of similar reports about regional practice variation among RTs. The factors that contribute to regional practice variation among RTs in home oxygen evaluation deserve further investigation.

There appears to be no clear explanation as to why RTs are not routinely involved in selecting home oxygen equipment. In clinical practice, RTs generally work under the direction of and together with physicians to make decisions regarding home oxygen therapy and equipment. This might explain the low reported rate of RTs as the only decisionmakers for home oxygen equipment in our study. Interestingly, durable medical equipment providers were reported to be involved in selecting home oxygen equipment in nearly $20 \%$ of patients, which raises concern for possible conflicts of interest.

The lack of routine RT participation in the selection of home oxygen equipment may also be due to the lack of defined roles and responsibilities. There is increasing recognition about the importance of multidisciplinary teams, including RTs, physicians, nurses, and physical therapists, to liberate patients from ventilators. ${ }^{15,16}$ This is supported by the CMS advisory to hospitals in $\S 482.43$ (b)(2) of its Interpretive Guidelines, which states that a "well-designed 


\section{Home OXYgEn Evaluation by RTs}

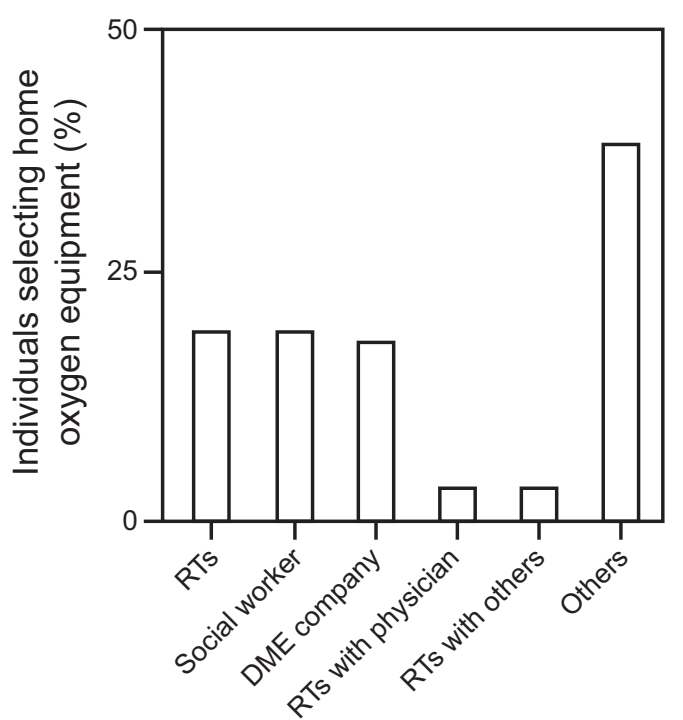

Fig. 3. The selection of home oxygen equipment involved RTs in 25\% (19\% RTs, 3\% RTs and physicians, 3\% RTs and others). Others included alone or in combination with nurses, discharge planners, case managers, social workers, DME companies, and patients. $12.8 \%(63 / 490)$ of the answers were missing. RT= respiratory therapist; $\mathrm{DME}=$ durable medical equipment.

discharge planning evaluation uses a multidisciplinary approach [...] to ensure that all of the patient's postdischarge needs are identified" (https://www.cms.gov, Accessed July 5, 2020).

This study has 2 strengths. This is the first study to date to examine the role of RTs in the evaluation for home oxygen before discharge in patients hospitalized for COPD. The RTs in our study were recruited from almost all states in the United States and are comparable with those described in the AARC Human Resources study with regard to surveyed characteristics, therefore increasing the likelihood that our findings reflect national practice patterns of RTs who are members of the AARC. ${ }^{12}$ This study has some limitations. Respondents were all members of the AARC and self-selected to participate, and they may differ in some important ways from non-respondents or from RTs who are not members of the AARC. The data were selfreported, so we cannot exclude the possibility of social desirability bias resulting in an overestimate of reporting consistent evaluation for home oxygen. Our report also did not examine barriers and facilitators in assessing the need for home oxygen in patients hospitalized for COPD exacerbations, post-hospital care practices, nor access to home oxygen equipment.

\section{Conclusions}

Our results indicate that RTs do not consistently evaluate for home oxygen in patients hospitalized for COPD exacerbations and are rarely involved in selecting home oxygen equipment. Our study provides information that could contribute to future interventions incorporating RTs in the assessment for home oxygen needs prior to hospital discharge. Further research is needed to study multidisciplinary interventions and successful initiation of home oxygen therapy incorporating RTs at the time of the transition from hospital to home.

\section{ACKNOWLEDGMENTS}

The authors thank Hannah Nguyen MPH, and Richard Gallardo III MPH, for their assistance in data analysis as well as the staff at the executive office of the American Association for Respiratory Care for their assistance in conducting the study.

\section{REFERENCES}

1. Chaney JC, Jones K, Grathwohl K, Olivier KN. Implementation of an oxygen therapy clinic to manage users of long-term oxygen therapy. Chest 2002;122(5):1661-1667.

2. Christopher KL, Porte P. Long-term oxygen therapy. Chest 2011;139 (2):430-434

3. Zaidi F, Lee RS, Buchcic BA, Bracken NE, Jaffe HA, Joo M, et al. Evaluation and documentation of supplemental oxygen requirements is rarely performed in patients hospitalized with COPD. Chronic Obstr Pulm Dis 2017;4(4):287-296.

4. Jacobs SS, Lindell KO, Collins EG, Garvey CM, Hernandez C, McLaughlin S, et al. Patient perceptions of the adequacy of supplemental oxygen therapy. Results of the American Thoracic Society Nursing Assembly Oxygen Working Group Survey. Ann Am Thorac Soc 2018;15(1):24-32.

5. Kallstrom TJ, on behalf of American Association for Respiratory Care (AARC). AARC Clinical Practice Guideline: Oxygen Therapy for Adults in the Acute Care Facility - 2002 revision and update. Respir Care 2002;47(6):717-720.

6. Sculley J, Corbridge S, Prieto-Centurion V, Kallstrom T, Lewarski J, Tan AM, Krishnan JA. Home oxygen therapy for patients with COPD: time for a reboot. Respir Care 2019;64(12):1574-1585.

7. Stelfox HT, Hess DR, Schmidt UH. A North American survey of respiratory therapist and physician tracheostomy decannulation practices. Respir Care 2009;54(12):1658-1664.

8. Leddy R, Wilkinson JM. Endotracheal suctioning practices of nurses and respiratory therapists: how well do they align with clinical practice guidelines? Can J Respir Ther 2015;51(3):60-64.

9. Long term domiciliary oxygen therapy in chronic hypoxic cor pulmonale complicating chronic bronchitis and emphysema. Report of the Medical Research Council Working Party. Lancet 1981;1(8222):681686.

10. Nocturnal Oxygen Therapy Trial Group. Continuous or nocturnal oxygen therapy in hypoxemic chronic obstructive lung disease: a clinical trial. Ann Intern Med 1980;93(3):391-398.

11. Eaton T, Garrett JE, Young P, Fergusson W, Kolbe J, Rudkin S, Whyte K. Ambulatory oxygen improves quality of life of COPD patients: a randomised controlled study. Eur Respir J 2002;20(2):306312.

12. O'Donnell DE, D'Arsigny C, Webb KA. Effects of hyperoxia on ventilatory limitation during exercise in advanced chronic obstructive pulmonary disease. Am J Respir Crit Care Med 2001;163(4):892-898.

13. Davidson AC, Leach R, George RJ, Geddes DM. Supplemental oxygen and exercise ability in chronic obstructive airways disease. Thorax 1988;43(12):965-971. 


\section{Home OXYgEn Evaluation by RTs}

14. Krishnan JA, Bracken NE, Cerretta S, Gonzalez V, Grabarek M, Holm $\mathrm{K}$, et al. What oxygen equipment do patients with COPD have at home? Ancillary results of the PELICAN Study (abstract). Am J Respir Crit Care Med 2017;195:A4715.

15. Ouellette DR, Patel S, Girard TD, Morris PE, Schmidt GA, Truwit JD, et al. Liberation from mechanical ventilation in critically ill adults: an official American College of Chest Physicians/American Thoracic
Society Clinical Practice Guideline: inspiratory pressure augmentation during spontaneous breathing trials, protocols minimizing sedation, and noninvasive ventilation immediately after extubation. Chest 2017;151(1):166-180.

16. Engel HJ, Needham DM, Morris PE, Gropper MA. ICU early mobilization: from recommendation to implementation at three medical centers. Crit Care Med 2013;41(9 Suppl 1):S69-S80.

This article is approved for Continuing Respiratory Care Education credit. For information and to obtain your CRCE

(free to AARC members) visit

www.rcjournal.com 